\title{
Niejednoznaczność wymiarów doświadczenia z marką na przykładzie badań empirycznych w warunkach polskich
}

\section{Ambiguity of the dimensions of brand experience on an example of empirical studies in Polish consumers conditions}

\author{
mgr Damian Badzmirowski \\ ORCID 0000-0003-2170-9281 \\ mgr Oskar Uchański \\ ORCID 0000-0002-4455-5070 \\ Centrum Badań Rynkowych i Spotecznych Sp. z o.o. \\ info@cebris.pl
}

\begin{abstract}
W artykule autorzy prezentują wyniki badań ilościowych CATI na temat doświadczeń z marką, mających na celu weryfikację istnienia i znaczenia wymiarów doświadczeń wśród klientów korzystających z produktów w obrębie wybranych kategorii produktowych: kawa, czekolada, perfumy i smartfony. Zwracają uwagę na wymiary doświadczenia z marką, które nie są sobie równoważne. Zaproponowane wymiary posiadają rożne wartości w zakresie wyjaśniania doświadczenia z marką $\mathrm{i}$ wchodzą w skład rożnych czynników stojących za obserwowaną zmiennością.
\end{abstract}

Keywords

doświadczenia z marką, doświadczenia konsumenckie, badania marketingowe, badanie marki, teoria marki

In this article, the authors present the results of quantitative research (CATI) on brand experience, aimed at verifying the existence and significance of experience dimensions among customers using products within selected product categories: coffee, chocolate, perfumes and smartphones. The authors notice dimensions of experience with the brand, which are not equivalent. The dimensions proposed by the authors have different values in terms of explaining the experience with the brand and are included in the various factors behind the observed variability.

Slowa kluczowe

brand experience, consumer experience, marketing research, brand research, brand theory

JEL: M310

Str. $38-46$

\section{Bibliografia}

Aaker, D. A. (1996). Building Strong Brands. New York: The Free Press.

Aaker, J. L. (1997). Dimensions of brand personality. Journal of Marketing Research, XXXIV, $347-356$. https://doi.org/10.1177/002224379703400304

Antonetti, P. i Maklan, S. (2014). Feelings that make a difference: How guilt and pride convince consumers of the effectiveness of sustainable consumption choices. Journal of Business Ethics, 124(1), 117-134. https://doi.org/ 10.1007/s10551-013-1841-9

Babin, B. J., i Griffin, M. (1998). The nature of satisfaction: An updated examination and analysis. Journal of Business Research, 41(2), 127-136. https://doi.org/10.1016/S0148-2963(97)00001-5 
Bloch, P. H., Brunel, F. F. i Arnold, T. J. (2003). Individual differences in the centrality of visual product aesthetics: concept and measurement. Journal of Consumer Research, 29, 551-565. https://doi.org/10.1086/346250

Bone, P. F. i Ellen, P. S. (1999). Scents in the marketplace: Explaining a Fraction of olfaction. Journal of Retailing, 75(2), 243-262. https://doi.org/10.1016/S0022-4359(99)00007-X

Brakus, J. J., Schmitt, B. H. i Zarantonello, L. (2009). Brand experience: What is it? How is it measured? Does it affect loyalty? Journal of Marketing, 73, 51-68. https://doi.org/10.1509/jmkg.73.3.52

Ciobanu, V. i Boglut, A. (2014). Reasons for buiyng a product. Research Journal of Agricultural Science, 46(1), 132-138.

Damasio, A. (1999). The feeling of what happens: Body and emotion in the making of consciousness. New York: Hartcourt.

Davis, J. A. (2007). Measuring Marketing: 103 Key Metrics Every Marketer Needs. Singapore: John Wiley \& Sons (Asie).

Dziewanowska, K. (2013). Nowe oblicze marketingu — koncepcja marketingu doświadczeń. Marketing i Rynek, (1), 16-24.

Dziewanowska, K. i Kacprzak, A. (2016). Działania z zakresu marketingu doświadczeń w oczach polskich konsumentów wyniki badań jakościowych. Studia Ekonomiczne. Zeszyty Naukowe Uniwersytetu Ekonomicznego w Katowicach, 255, $18-27$.

Farlex Inc. (2009). Purchase frequency. W: Farlex Financial Dictionary. Farlex.

Festinger, L. (2007). Teoria dysonansu poznawczego. Warszawa: Wyd. Naukowe PWN.

Fourie, J. (2017). How social status drives our consumption - and inequality. Finweek. Pozyskano z https://www.fin24.com/Finweek/Opinion/how-socialstatus-drives-our-consumption-and-inequality-20170608

Gentile, C., Spiller, N. i Noci, G. (2007). How to Sustain Customer Experience: An Overview of Experience Components that Co-create Value with the Customer. European Management Journal, 25(5).

Gitomer, J. (2008a). Find positives with customers, not negatives. Des Moines Business Record. Pozyskano z http://search.ebscohost. com/login.aspx?direct=true \&db=f5h\&AN=31853041\&site=ehost-live

Gitomer, J. (2008b). Don't sell by trying to find the customer's pain. The Central New York Business Journal. Pozyskano z http://search.ebscohost.com/login.aspx?direct=true \&db=f5h\&AN=39236043\& site=ehost-live

Hilton, K. (2015). Psychology the science of sensory marketing. Harvard Business Review, 93(3), $28-30$.

Hirschman, E. C. i Holbrook, M. B. (1982). Hedonic Consumption: Emerging Concepts, Methods and Propositions. Journal of Marketing, 46(3), 92-101. https://doi.org/10.1177/002224298204600314

Hoch, S. J. (2002). Product experience is seductive. Journal of Consumer Research, 29, 448-454. https://doi.org/ $10.1086 / 344422$

Hulten, B., Broweus, N. i van Dijk, M. (2011). Marketing sensoryczny. Warszawa: PWE.

Iftikhar, M., Hussain, M. F., Kahn, Z. A. i Iiyas, S. (2013). Social class is a myth or reality in buying behavior. African Journal of Business Management, 7(9), 713-718.

Kahn, B. E., i Isen, A. M. (1993). The influence of positive affect on variety seeking among safe, enjoyable products. Journal of Consumer Research, 20, 257-270. https://doi.org/10.1086/209347

Kapferer, J. -N. (1986). Beyond positioning, retailers identity. Esomar Seminar Proceedings, (4-6), 167-176.

Kapferer, J. -N. (2008). The New Strategic Brand Management. London: Kogan Page.

Keller, K. L. (1993). Conceptualizing, measuring, and managing customer-based brand equity. Journal of Marketing, 57, 122. https://doi.org/10.1177/002224299305700101

Keller, K. L. (2003). Strategic Brand Management: Building, Measuring and Managing Brand Equity. Upper Saddle River: Pearson.

Knapp, D. E. (2008). The Brand Promise. New York: McGraw Hill.

Kohli, C. i Leuthesser, L. (2001). Brand equity: Capitalizing on intellectual capital. Ivey Business Journal, 65(4), 74.

Kotler, P. T. (1991). Marketing Management: Analysis, Planning, and Control. Englewood Cliffs: Prentice-Hall.

Kotler, P. T. i Keller, K. L. (2009). Marketing Management. Upper Saddle River: Pearson-Prentice Hall.

Kumar Dawn, S. (2014). Personalised Marketing: concepts and framework. Productivity, 54(4), 370-377.

Lageat, T., Czellar, S. i Laurent, G. (2003). Hedonic attributes engineering of luxury: To generate perceptions of an everyday consumer sound perception. Marketing Letters, 14(2), 97-109. https://doi.org/10.1023/A:1025462901401

Liang, B. i He, Y. (2012). The effect of culture on consumer choice: The need for conformity vs. the need for uniqueness. International Journal of Consumer Studies, 36(3), 352-359. https://doi.org/10.1111/j.1470-6431.2011.01013.x

Maslow, A. H. (1954). Motivation and Personality. New York: Harper.

McLellan, D. (2014). Find your sweet spot. Des Moines Business Record, 32(43).

Mittal, V. i Kamakura, W. A. (2001). Satisfaction, repurchase Intent, and repurchase behavior - investinating the moderating effect of consumer characteristics. Journal of Marketing Research, XXXVIII, 131-142. https://doi.org/ 10.1509/jmkr.38.1.131.18832

Nadeem, M. M. (2007). Post-purchase dissonance: The wisdom of the "repeat" purchases. Journal of Globa Business Issues, 1(2), 183-193.

Newman, J. (1957). New insight, new progress, for marketing. Harvard Business Review, 35(6), 95-102. 
Oliver, R. L. (1999). Whence consumer loyalty? Journal of Marketing, 63, 33-44. https://doi.org/10.1177/ 00222429990634 s 105

Oliver, R. L., Rust, R. T. i Varki, S. (1997). Customer delight: Foundations, findings, and managerial insight. Journal of Retailing, 73(3), 311-336. https://doi.org/10.1016/S0022-4359(97)90021-X

Paasovaara, R., Luomala, H.T., Pohjanheimo, T. i Sandell, M. (2012). Understanding consumers' brand-induced food taste perception: A comparison of "brand familiarity" — and "consumer value — brand symbolism (in)congruity" accounts. Journal of Consumer Behaviour, 11, 11-20. https://doi.org/10.1002/cb.356

Pham, M. T. (2012). The neglected dimension of affective states: New findings on the effects of stress, relaxation, anxiety, and arousal, on consumer behavior. Advances in Consumer Research, 38, 119-121.

Ries, A. i Trout, J. (1986). Positioning: The Battle for Your Mind. New York: McGraw-Hill.

Romaniuk, J., Wight, S. i Faulkner, M. (2017). Brand awareness: Revisiting an old metric for a new world. Journal of Product \& Brand Management, 26(5), 469-476. https://doi.org/10.1108/JPBM-06-2016-1242

Rossiter, J. R., i Larry, P. (1987). Advertising and Promotion Management. New York: McGraw-Hill.

Russo, F. (2018). Our Stuff, Ourselves. Scientific American, 318(5). https://doi.org/10.1038/scientificamerican0518-66

Rucker, D. D. i Galinsky, A. D. (2008). Desire to acquire: Powerlessness and compensatory consumption. Journal of Consumer Research, 35(2), 257-267. https://doi.org/10.1086/588569

Schmitt, B. H. (1999). Experiential marketing. Journal of Marketing Management, 15, 53-67. https://doi.org/10.1362/ 026725799784870496

Shen, A. (2014). Recommendations as personalized marketing: Insights from customer experiences. Journal of Services Marketing, 28(5), 414-427. https://doi.org/10.1108/JSM-04-2013-0083

Shultz, S., i Hand, M. W. (2015). Usability: A concept analysis. Journal of Theory Construction \& Testing, 19(2), 65-70.

Silvia, P. J. (2006). Exploring the Psychology of Interest. Oxford University Press. https://doi.org/10.1093/ acprof:oso/9780195158557.001.0001

Skorek, M. (2016). Attitudes of Polish consumers toward experiential marketing. Journal of Management and Business Administration. Central Europe, 24(4), 109-124. https://doi.org/10.7206/jmba.ce.2450-7814.185

Skowronek, I. (2011). Oddziaływanie zapachem jako forma marketingu sensorycznego. Marketing i Rynek, (1), 6-11.

Strombeck, S. D. i Shu, S.-T. (2016). The pursuit of social recognition through luxury service brands. Services Marketing Quarterly, 37(4), 241-254. https://doi.org/10.1080/15332969.2016.1217682

Szromek, A. R. (2015). Zjawisko dysonansu i konsonansu poznawczego w zachowaniach konsumentów produktu turystycznego - wprowadzenie do zagadnienia. Prace Naukowe Uniwersytetu Ekonomicznego we Wrocławiu, 379, 348-355. https://doi.org/10.15611/pn.2015.379.33

Tantry, S. (2016). Making personalized marketing work. Harvard Business Review. Pozyskano z https://hbr.org/ 2016/02/making-personalized-marketingwork

Waszkiewicz-Raviv, A. (2015). Wizualna konsumpcja a komunikacja wizualna. Perswazyjność obrazu w ramach współczesnej komunikacji marketingowej. Prace Naukowe Uniwersytetu Ekonomicznego we Wrocławiu, 414, 42-51.

Williams, L. E. i Bargh, J. A. (2008). Keeping one's distance: The influence of spatial distance cues on affect and evaluation. Psychological Science, 19(3), 302-308. https://doi.org/10.1111/j.1467-9280.2008.02084.x

Zawadzka, A. M. (2007). Dlaczego przywiązujemy się do marki? Gdańsk: GWP.

Zhong, J. Y. i Mitchell, V. W. (2012). Does consumer well-being affect hedonic consumption? Psychology and Marketing, 29(8), 583-594. https://doi.org/10.1002/mar.20545 